\title{
Is there a difference between normotensive and hypertensive patients in terms of blood parameters and cardiovascular diseases?
}

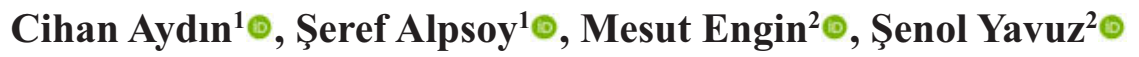 \\ ${ }^{1}$ Department of Cardiology, Namık Kemal University, Faculty of Medicine, Tekirdăg, Turkey \\ ${ }^{2}$ Department of Cardiovascular Surgery, University of Health Sciences, , Bursa Yüksek Ihtisas Training and Research Hospital, Bursa, \\ Turkey
}

\section{ABSTRACT}

Objectives: It has been stated in various studies that there is a difference in some blood parameters between hypertensive and normotensive patients for a long time. Mean platelet volume (MPV), and red blood cell distribution width (RDW), have been studied in hypertensive patient groups in many studies. Hypertension is a classic risk factor for ischemic stroke and myocardial ischemia, as known. In our study, we examined whether there was a difference between hypertensive patient groups and normotensive patients in terms of blood parameters such as MPV and RDW, and the incidence of stroke and myocardial infarction.

Methods: Blood samples and twenty-four-hour ambulatory blood pressure monitoring (ABPM) results of 552 patients admitted to our outpatient clinic with a pre-diagnosis of hypertension were retrospectively analyzed. According to ABPM results, we divided the study participants into four groups; dippers, non-dippers, extreme dippers, and normotansives. Complete blood count and biochemical test results were found in the database of our hospital for all patients and differences between groups were investigated.

Results: One hundred seventy three normotensives (Group 1) (mean age, $47.4 \pm 15.4$ years), 210 non-dippers (Group 2) (mean age, $53.8 \pm 15.8$ years), 67 extreme dippers (Group 3) (mean age, $49.1 \pm 15.9$ years) and 102 dippers (Group 4) (mean age, $52.2 \pm 12.5$ years). Daytime mean systolic blood pressure (SBP) and diastolic blood pressure (DBP) and night-time mean SBP and DBP were significantly different in groups (Group 1: 117 [90-193] mmHg and 71 [55-87] mmHg; Group 2: 137 [107-188] mmHg and 83 [107-188] mmHg; Group 3: 143 [115-193] mmHg and 88 [56-122] mmHg; and Group 4: 140.5 [116-173] mmHg and 76 [55-124] mmHg), $p<0.001$; respectively. MPV and RDW levels were different in all four groups $(p<0.001)$. We found a significant difference in the rates of stroke and coronary artery disease between the four groups $(p=0.018$ and $p=0.002$, respectively). In the ROC curve analysis MPV had sensitivity of $\% 77.8$ and specificity of $78.1 \%$ for stroke when the cut-off value MPV was 9.25 (Area under curve: $0.808,95 \%$ confidence interval: 0.726 $0.889, p<0.001)$.

Conclusions: In our study, MPV and RDW levels and the rates of stroke and cardiovascular disease were significantly higher in non-dipper patients compared to other groups.

Keywords: Hypertension, platelet, inflammation, complications

How to cite this article: Aydin C, Alpsoy S, Engin M, Yavuz S. Is there a difference between normotensive and hypertensive patients in terms of blood parameters and cardiovascular diseases?. Eur Res J 2021;7(6):594-600. DOI: 10.18621/eurj.990051

Address for correspondence: Cihan Aydın, MD., Namık Kemal University, Faculty of Medicine, Department of Cardiology, Namık Kemal Kampüs Street,59030 Tekirdağ,Turkey.E-mail: drcihanaydin@hotmail.com,Tel: +902822500000 
A rterial blood pressure has a circadian rhythm that affects cardiovascular outcomes [1]. In a normal population, average night-time systolic and diastolic blood pressure (BP) decrease \%10-\%20 compared to daytime BP. The dipping pattern is a $10 \%$ to $20 \%$ decrease in night-time mean systolic or diastolic BP relative to mean daytime BP. This drop rate is below $10 \%$ in the non-dipper group. The average night BP drop is over $20 \%$ in extreme dippers [2]. Previous studies demonstrated that as in the non-dipper group, the frequency of cardiovascular and cerebrovascular events increases in the extreme dipper group. Especially silent cerebral infarct rate increases in this group [3].

Red cell distribution width (RDW) increases due to increased erythrocyte destruction or ineffective erythropoiesis. Changes in the RDW interval may predict other cardiovascular disease outcomes $[4,5]$. Previous studies demonstrated that hypertensive patients might have higher RDW levels according to patients with normal BP. In addition, in some studies, RDW levels were higher in the non-dipper group compared to the dipper group [6]. Mean platelet volume (MPV) is an indicator of platelet activation and platelet size, which is an independent risk factor for hypertension, myocardial infarction, and stroke $[7,8]$. It is known that active large platelets containing denser granules are metabolically and enzymatically more active in triggering thrombosis than small ones [9].

In our study, we planned to compare MPV and RDW levels and the frequency of stroke and cardiovascular disease in between dippers, non-dippers, extreme-dippers, and normotensives.

\section{METHODS}

\section{Study Population}

The data and ambulatory blood pressure monitoring (ABPM) results of 552 patients over the age of 18 who were diagnosed with hypertension between January 2019 and June 2020 in our outpatient clinic were evaluated retrospectively. The patients were divided into four groups (dippers, non-dippers, normotensives, and extreme dippers) based on the results of ABPM. The exclusion criteria of this study were as follows; secondary hypertension, congestive heart failure, recent myocardial infarction (MI), peripheral vascular disease, valvular disease, having a history of metallic prosthetic valve surgery, angina pectoris, chronic renal failure (serum creatinine $>1.5 \mathrm{mg} / \mathrm{dl}$ ), chronic liver diseases, thromboembolic disorders, hematological disorders, acute or chronic infections. Patients under antiplatelet and warfarin treatment were also excluded from the study. Informed consent was obtained from all participants, and the study protocol was approved by the Namık Kemal University ethics committee.

\section{Ambulatory Blood Pressure Monitoring}

Hypertension was defined as the mean of three different BP measurements above 140/90 $\mathrm{mmHg}$. In ABPM average $\mathrm{SBP}>135 \mathrm{mmHg}, \mathrm{DBP}>85 \mathrm{mmHg}$ at daytime (awake), average $\mathrm{SBP}>120 \mathrm{mmHg}$, DBP $>70 \mathrm{mmHg}$ at nighttime (asleep), or in average 24hour measurements SBP $>130 \mathrm{mmHg}$, DBP $>80$ $\mathrm{mmHg}$ defined as hypertension. Patients with a mean of three measurements below 140/90 in-office BP measurements and those with BP measurements below the above-mentioned values in ABPM were considered normotensive.

Blood pressure, heart rate was measured and recorded automatically by the ABPM system. (Darwin Professional Medilog; Schiller BR-102, Switzerland). The recorder was programmed to record at 30-minute intervals during the day (7:00 am-10:00 pm) and 1hour intervals during the night (10:00 pm-7:00 am). Patients were subclassified according to the percentage difference between day and night systolic blood pressure (SBP) and diastolic blood pressure (DBP). Average nighttime SBP and DBP decrease \%10-\%20 compared to daytime BP. This group was named as dipper (Group 4). One hundred two patients who met this criterion were included in this group. The group with a decrease of $20 \%$ or more in systolic or diastolic $\mathrm{BP}$ at night compared to the daytime was named as extreme-dipper (Group 3). Sixty-seven patients were in the extreme dipper group. The group in which SBP or DBP at night decreased by 10 percent or less compared to daytime was named as non-dipper (Group 2). Two hundred ten patients were in this group. There were 173 normotensive patients as a control group (Group 1).

Baseline demographic data, current medications, and blood chemistry test results were collected from their medical records in our hospital. Blood samples were drawn from the antecubital vein between 08:00 am and 10:00 am after overnight fasting. EDTA 
(dipotassium ethylendinitrotetraacetic acid) containing tubes were used for blood samples.

\section{Statistical Analysis}

SPSS for Windows Version 22, 0 (SPSS Inc., IL, USA) was used for all statistical analysis. Continuous variables were expressed as mean \pm standard deviation (SD) and categorical variables were expressed as per- centage. Whether the parameters conformed to the normal distribution was evaluated with the Kolmogorov Smirnov test. In statistical parameters such as RDW, MVP with normal distribution One-way ANOVA test was used for 4 groups. In post hoc analysis, parameters with equality of variance according to Levene's test were evaluated with Tukey's test, and parameters without equality of variance were evalu-

Table 1. Baseline characteristics of the patient groups

\begin{tabular}{|c|c|c|c|c|c|c|}
\hline Variables & $\begin{array}{l}\text { GROUP 1 } \\
(\mathrm{n}=173)\end{array}$ & $\begin{array}{c}\text { GROUP } 2 \\
(n=210)\end{array}$ & $\begin{array}{l}\text { GROUP } 3 \\
(n=67)\end{array}$ & $\begin{array}{c}\text { GROUP } 4 \\
(n=102)\end{array}$ & $\begin{array}{c}\text { Total } \\
(\mathbf{n}=\mathbf{5 5 2})\end{array}$ & $p$ value \\
\hline Age (years) & $47.4 \pm 15.4$ & $53.8 \pm 15.8$ & $49.1 \pm 15.9$ & $52.2 \pm 12.5$ & $50.9 \pm 15.3$ & $<0.001^{\mathrm{c}}$ \\
\hline Male, n (\%) & $49(28.3 \%)$ & $99(47.1 \%)$ & $26(38.8 \%)$ & $47(46.1 \%)$ & $221(40 \%)$ & $0.001^{\mathrm{a}}$ \\
\hline Female, n (\%) & $124(71.7 \%)$ & $111(52.9 \%)$ & $41(61.2 \%)$ & $55(53.9 \%)$ & $331(60 \%)$ & $0.001^{\mathrm{a}}$ \\
\hline CAD, n (\%) & $12(6.9 \%)$ & $34(16.2 \%)$ & $2(3 \%)$ & $16(15.7 \%)$ & $64(11.6 \%)$ & $0.002^{\mathrm{a}}$ \\
\hline Stroke, n (\%) & $9(5.2 \%)$ & $27(12.9 \%)$ & $4(6 \%)$ & $5(4.9 \%)$ & $45(8.2 \%)$ & $0.018^{\mathrm{a}}$ \\
\hline $\mathrm{DM}, \mathrm{n}(\%)$ & $28(16.2 \%)$ & $48(22.9 \%)$ & $12(17.9 \%)$ & $23(22.5 \%)$ & $111(20.1 \%)$ & $0.358^{\mathrm{a}}$ \\
\hline Glucose, mg/dl & $100(75.6-176)$ & $101.65(78.6-592)$ & $99(76-264)$ & $102(76-438)$ & $95.9(75.6-592)$ & $0.060^{\mathrm{b}}$ \\
\hline Hemoglobin, gr/dL & $13.19 \pm 1.49$ & $13.48 \pm 1.73$ & $13.3 \pm 1.52$ & $13.87 \pm 1.56$ & $13.47 \pm 1.51$ & $0.070^{c}$ \\
\hline Hematocrit, \% & $40.1 \pm 5.85$ & $406 \pm 4.84$ & $41.04 \pm 4.37$ & $41.3 \pm 4.52$ & $40.76 \pm 4.89$ & $0.236^{\mathrm{c}}$ \\
\hline \multicolumn{7}{|l|}{ Medical Treatment } \\
\hline Beta blocker, n (\%) & $62(35.8 \%)$ & $134(63.8 \%)$ & $36(53.7 \%)$ & $60(58.8 \%)$ & $292(52.9 \%)$ & $<0.001^{\text {a }}$ \\
\hline Ca-channel blocker, n (\%) & $26(15 \%)$ & $71(33.8 \%)$ & $18(27.5 \%)$ & $28(27.5 \%)$ & $143(25.9 \%)$ & $0.001^{\mathrm{a}}$ \\
\hline ACE-I/ARB, n (\%) & $62(35.8 \%)$ & $134(63.8 \%)$ & $36(53.7 \%)$ & $60(58.8 \%)$ & $292(52.9 \%)$ & $<0.001^{\mathrm{a}}$ \\
\hline Diuretic, n (\%) & $40(23.1 \%)$ & $95(45.2 \%)$ & $26(38.8 \%)$ & $43(42.2 \%)$ & $204(37 \%)$ & $<0.001^{\mathrm{a}}$ \\
\hline $\mathrm{OAD}, \mathrm{n}(\%)$ & $25(14.5 \%)$ & $41(19.5 \%)$ & $8(11.9 \%)$ & $23(22.5 \%)$ & $97(17.6 \%)$ & $0.177^{\mathrm{a}}$ \\
\hline Insulin, $\mathrm{n}(\%)$ & $6(3.5 \%)$ & $9(4.3 \%)$ & $2(3 \%)$ & $5(4.9 \%)$ & $22(4 \%)$ & $0.903^{\mathrm{a}}$ \\
\hline \multicolumn{7}{|l|}{$\begin{array}{l}\text { Ambulatory blood pressure } \\
\text { data }\end{array}$} \\
\hline $\begin{array}{l}24 \text { hours mean SBP } \\
(\mathrm{mmHg})\end{array}$ & $\begin{array}{c}115 \\
(90-132)\end{array}$ & $\begin{array}{c}137.5 \\
(107-184)\end{array}$ & $\begin{array}{c}135 \\
(111-189)\end{array}$ & $\begin{array}{c}136.5 \\
(111-169)\end{array}$ & $\begin{array}{c}123 \\
(90-189)\end{array}$ & $<0.001^{b}$ \\
\hline $\begin{array}{l}24 \text { hour mean DBP } \\
(\mathrm{mmHg})\end{array}$ & $\begin{array}{c}69 \\
(56-83)\end{array}$ & $\begin{array}{c}81.5 \\
(61-122)\end{array}$ & $\begin{array}{c}81 \\
(52-109)\end{array}$ & $\begin{array}{c}83 \\
(56-105)\end{array}$ & $\begin{array}{c}74 \\
(52-122)\end{array}$ & $<0.001^{\text {b }}$ \\
\hline 24 hour mean HR beat/min & $73.9 \pm 8.9$ & $74.8 \pm 9.9$ & $74.2 \pm 8.3$ & $74.9 \pm 9.2$ & $74.5 \pm 9.3$ & $0.762^{\mathrm{c}}$ \\
\hline $\begin{array}{l}\text { Daytime mean SBP } \\
(\mathrm{mmHg})\end{array}$ & $\begin{array}{c}117 \\
(90-193)\end{array}$ & $\begin{array}{c}137 \\
(107-188)\end{array}$ & $\begin{array}{c}143 \\
(115-193)\end{array}$ & $\begin{array}{c}140,5 \\
(116-173)\end{array}$ & $\begin{array}{c}125 \\
(90-193)\end{array}$ & $<\mathbf{0 . 0 0 1}^{\mathrm{b}}$ \\
\hline $\begin{array}{l}\text { Daytime mean DBP } \\
(\mathrm{mmHg})\end{array}$ & $\begin{array}{c}71 \\
(55-87)\end{array}$ & $\begin{array}{c}83 \\
(60-124)\end{array}$ & $\begin{array}{c}88 \\
(56-122)\end{array}$ & $\begin{array}{c}87 \\
(58-111)\end{array}$ & $\begin{array}{c}76 \\
(55-124)\end{array}$ & $<0.001 b$ \\
\hline $\begin{array}{l}\text { Daytime mean HR } \\
\text { beat/min }\end{array}$ & $\begin{array}{c}76 \\
(47-108)\end{array}$ & $\begin{array}{c}78 \\
(50-111)\end{array}$ & $\begin{array}{c}77 \\
(60-104)\end{array}$ & $\begin{array}{c}79 \\
(55-98)\end{array}$ & $\begin{array}{c}73,5 \\
(47-111)\end{array}$ & $0.743^{\mathrm{b}}$ \\
\hline $\begin{array}{l}\text { Night-time mean SBP } \\
(\mathrm{mmHg})\end{array}$ & $109.4 \pm 8.6$ & $139.5 \pm 17$ & $123.5 \pm 16.4$ & $129.8 \pm 11.4$ & $126.3 \pm 18.7$ & $<0.001^{\mathrm{c}}$ \\
\hline $\begin{array}{l}\text { Night-time mean DBP } \\
(\mathrm{mmHg})\end{array}$ & $\begin{array}{c}64 \\
(49-78)\end{array}$ & $\begin{array}{c}80 \\
(58-120)\end{array}$ & $\begin{array}{c}70 \\
(45-90)\end{array}$ & $\begin{array}{c}76 \\
(52-95)\end{array}$ & $\begin{array}{c}68 \\
(45-120)\end{array}$ & $<\mathbf{0 . 0 0 1}^{\mathrm{b}}$ \\
\hline $\begin{array}{l}\text { Night-time mean HR } \\
\text { beat/min }\end{array}$ & $\begin{array}{c}67 \\
(37-95)\end{array}$ & $\begin{array}{c}70 \\
(47-102)\end{array}$ & $\begin{array}{c}68 \\
(53-91)\end{array}$ & $\begin{array}{c}68 \\
(50-95)\end{array}$ & $\begin{array}{c}64 \\
(37-102)\end{array}$ & $0.132^{\mathrm{b}}$ \\
\hline $\begin{array}{l}\text { Daytime mean arterial } \\
\text { pressure }(\mathrm{mmHg})\end{array}$ & $\begin{array}{c}87 \\
(48.67-101.67)\end{array}$ & $\begin{array}{c}100.33 \\
(79.33-145 ., 33)\end{array}$ & $\begin{array}{c}105 \\
(91.67-145.67)\end{array}$ & $\begin{array}{c}105.15 \\
(84.7-131.7)\end{array}$ & $\begin{array}{c}93 \\
(48.67-145.67)\end{array}$ & $<0.001^{\text {b }}$ \\
\hline $\begin{array}{l}\text { Night-time mean arterial } \\
\text { pressure }(\mathrm{mm} \mathrm{Hg})\end{array}$ & $79.09 \pm 5.9$ & $101.1 \pm 11.9$ & $87.5 \pm 9.1$ & $94.4 \pm 7.5$ & $91.3 \pm 13.1$ & $<0.001^{\mathrm{c}}$ \\
\hline $\begin{array}{l}24 \text { hour mean arterial } \\
\text { pressure }(\mathrm{mmHg})\end{array}$ & $\begin{array}{c}83.6 \\
(68.67-97)\end{array}$ & $\begin{array}{c}99 \\
(82-142)\end{array}$ & $\begin{array}{c}97 \\
(85.33-135.67)\end{array}$ & $\begin{array}{c}101.5 \\
(80.7-126.3)\end{array}$ & $\begin{array}{c}90.67 \\
(68.67-142)\end{array}$ & $<0.001^{b}$ \\
\hline
\end{tabular}

$\mathrm{BMI}=$ Body mass index, $\mathrm{SBP}=$ Systolic blood pressure, $\mathrm{DBP}=$ Diastolic blood pressure, $\mathrm{DM}=$ Diabetes mellitus, HR $=$ Heart rate, $\mathrm{CAD}=$ Coronary artery disease, OAD $=$ Oral anti-diabetic. ${ }^{\mathrm{a} C h i}$ square test (percentage), ${ }^{\mathrm{b} K r u s k a l}$ Wallis (median [minimummaximum]) ${ }^{\mathrm{C}}$ One Way Anova (mean \pm standard deviation)

Group 1 = normotensives, Group 2 = non-dippers, Group 3 = extreme dippers, Group 4 = dippers 
ated with Tamhane's test.Data that did not conform to normal distribution were compared using the Kruskal Wallis test.Mann-Whitney U test was used in subgroup analysis among 4 groups for parameters with significant differences. Frequency data were compared using the chi-square test. The cut-off value for MPV was calculated by performing roc analysis in individuals with strokes. $P$-value less than 0.05 was accepted statistically significant.

\section{RESULTS}

The mean age of 552 patients was $50.9 \pm 15.3$ years and $221(40 \%)$ were males, $331(60 \%)$ were females. The patients were divided into 4 groups according to their 24-hour ambulatory BP measurements; Group 1: normotensives (mean age: $47.4 \pm 15.4$ years; 49 (28.3\%) males, 124 (71.1\%) females), Group 2 : non-dippers (mean age: $53.8 \pm 15.8$ years; $99(47.1 \%)$ males, 111 (52.9\%) females), Group 3: extreme dippers (mean age: $49.1 \pm 15.9$ years; $26(38.8 \%)$ males, $41(61.2 \%)$ females) and Group 4: dippers (mean age: $52.2 \pm 12.5$ years; $47(46.1 \%)$ males, $55(53.9 \%)$ females). The four groups were similar in terms of the number of diabetic patients $(p=0.358)$. There was no significant difference between the groups in terms of insulin and oral antidiabetic use $(p=0.903$ and $\mathrm{p}=$ 0.177 , respectively). Twenty-four-hours, daytime and night-time mean heart rates of patients were similar in the measurements of ambulatory BP ( $p=0.762, p=$ 0.743 and $p=0.132$, respectively). Their baseline characteristics were summarized in Table 1.

Subgroup analysis of patients characteristics between four groups were presented in Table 2. The mean age of the other three groups was higher than Group $1(p<0.001)$. Coronary artery disease rate was statistically similar in Group 3 and Group $4(p>0.05)$ but higher than normotensives ( $p=0.002$ ). The stroke rate was highest in Group 2, and it was observed at a statistically similar rate in the other groups $(p=0.018)$. Except for Group 1, the lipid levels of the patients in the three groups were similar. Compared with Group 1 in terms of total cholesterol, (TC) triglyceride and low-density lipoprotein cholestrerol (LDL-C) levels were higher in Group $2(p<0.005, p<0.001$ and $p<$ 0.05 respectively). Triglyiceride levels in Group 3 and LDL-C levels in Group 4 were higher than Group 1.
When we evaluated the biochemical parameters, the levels of MPW and RDW values were significantly higher in Group 2 ( $p<0.001$ for two values). These parameters were similar among other groups $(p>$ $0.05)$. There was no difference between the groups in terms of other complete blood parameters and biochemical parameters.

The highest use of BP medication was in the nondipper group $(p<0.001)$ and it was similar in Group 3 and Group $4(p>0.05)$. Daytime /Night-time mean DBP, Daytime/Night-time mean SBP, Day/Night-time mean arterial pressures were significantly different between groups. 24-hour mean SBP / DBP and 24-hour mean arterial pressure was similar in the 3 groups but higher than Group 1.

ROC curve analysis showed that MPV had a sensitivity of $77.8 \%$ and specificity of $78.1 \%$ for stroke in individuals who participated in the study when the cut- off value of MPV was $9.25 \mathrm{fl}(p<0.001)$ (Fig. 1).

\section{DISCUSSION}

In our study, we found higher levels of MPV and RDW showing platelet activation in the non-dipper group compared to the other groups. Also, the frequency of cerebrovascular and coronary artery disease was higher in the non-dipper group.

In the long term, hypertension is a risk factor that should be controlled, causing end-organ damage and poor cardiovascular and cerebrovascular events [10]. Especially the non-dipper pattern is associated with target organ damage and poor cardiovascular outcomes in many studies [11]. In a meta-analysis in which prospective studies were examined, the dipping pattern and the mean night/day BP ratio significantly predicted mortality and cardiovascular events [12].

High MPV and RDW levels, which shown to be associated with inflammation and platelet activation, have been found to increase cardiovascular disease and mortality in hypertensive patients [13]. The underlying causes of high RDW levels are chronic inflammation, erythropoiesis disorders, increased erythrocyte destruction, and oxidative stress. RDW, a marker of inflammation, was found to be increased in non-dipper and dipper hypertensive patients compared to normotensive patients [14].

Ongoing oxidative stress and inflammation, in- 
Table 2. Subgroup analysis of patients characteristics between four groups

\begin{tabular}{|c|c|c|c|c|c|c|}
\hline Variables & $\begin{array}{c}\text { GROUP 1 } \\
(n=173)\end{array}$ & $\begin{array}{c}\text { GROUP } 2 \\
(n=210)\end{array}$ & $\begin{array}{l}\text { GROUP } 3 \\
(n=67)\end{array}$ & $\begin{array}{c}\text { GROUP } 4 \\
(n=102)\end{array}$ & $\begin{array}{c}\text { Total } \\
(n=552)\end{array}$ & $p$ value \\
\hline Age, (years) $)^{3 a}$ & $47.4 \pm 15.4$ & $53.8 \pm 15.8$ & $491 \pm 15.9$ & $52.2 \pm 12.5$ & $50.9 \pm 15.3$ & $<0.001^{a}$ \\
\hline $\mathrm{MPV}, \mathrm{fl}^{3 \mathrm{a}}$ & $8.52 \pm 0.8$ & $9.13 \pm 1.05$ & $8.74 \pm 0.78$ & $8.8 \pm 0.79$ & $8.83 \pm 0.95$ & $<0.001^{\mathrm{a}, \mathrm{d}, \mathrm{e}}$ \\
\hline $\mathrm{RDW}, \%^{3 \mathrm{a}}$ & $13.1 \pm 1.4$ & $13.8 \pm 1.39$ & $13.3 \pm 1.27$ & $13.01 \pm 1.2$ & $13.42 \pm 1.38$ & $<0.001^{\mathrm{a}, \mathrm{d}, \mathrm{e}}$ \\
\hline Hemoglobin, $g / \mathrm{dL}^{3 a}$ & $13.19 \pm 1.49$ & $13.48 \pm 1.73$ & $13.3 \pm 1.52$ & $13.87 \pm 1.56$ & $13.47 \pm 1.51$ & $0.008^{c}$ \\
\hline Platelet $\left(10^{3} / \mu \mathrm{L}\right)^{2}$ & $254(131-448)$ & $242(72-542)$ & $198(155.2-330)$ & $197.15(98.2-352)$ & $229(72-547)$ & 0.024 \\
\hline Creatinine $(\mathrm{mg} / \mathrm{dL})^{2}$ & $0.72(0.36-0.9)$ & $0.78(0.43-3.89)$ & $0.73(0.34-1.85)$ & $0.76(0.49-1.68)$ & $0.69(0.34-6.89)$ & $0.005^{\mathrm{a}}$ \\
\hline Total Cholesterol $(\mathrm{mg} / \mathrm{dL})^{2}$ & $185(59.5-354.4)$ & $201.4(98.9-357)$ & $198(155.2-330)$ & $197.15(98.2-352)$ & $179.5(59.5-357)$ & $0.003^{a, b, c}$ \\
\hline LDL-C $(\mathrm{mg} / \mathrm{dL})^{2}$ & $\begin{array}{c}106.78(19.8- \\
254.32)\end{array}$ & $120(35-232.7)$ & $120(68-220)$ & $117.71(31.96-256)$ & $\begin{array}{c}101.16(19.8- \\
256)\end{array}$ & $0.009^{a, b}$ \\
\hline HDL-C $(\mathrm{mg} / \mathrm{dL})^{2}$ & $48(21-125)$ & $45(20-97)$ & $48(32-84)$ & $49(30-106)$ & $41(20-125)$ & $0.015 a, d, e$ \\
\hline Triglyceride $(\mathrm{mg} / \mathrm{dL})^{2}$ & $\begin{array}{c}117.3(35.6- \\
1119)\end{array}$ & $149.75(47-650)$ & $136(50-478)$ & $120.4(45.7-526.2)$ & $105(35.6-1119)$ & $<0.001^{\mathrm{a}}$ \\
\hline $\mathrm{CAD}, \mathrm{n}(\%)^{1}$ & $8(4.62 \%)^{\mathrm{x}}$ & $34(16.2 \%)^{y}$ & $5(7.46 \%)^{z}$ & $7(6.8 \%)^{z}$ & $54(9.7 \%)$ & 0.002 \\
\hline Stroke, $\mathrm{n}(\%)^{1}$ & $9(5.2 \%)^{x}$ & $27(12.9 \%)^{y}$ & $4(6 \%)^{x}$ & $5(4.9 \%)^{x}$ & $45(8.2 \%)$ & 0.018 \\
\hline \multicolumn{7}{|l|}{ Gender $^{1}$} \\
\hline Male, $n(\%)^{1}$ & $49(28.3 \%)^{\mathrm{x}}$ & $99(47.1 \%)^{\mathrm{y}}$ & $26(38.8 \%)^{\mathrm{z}}$ & $47(46.1 \%)^{y}$ & $221(40 \%)$ & 0.001 \\
\hline Female, $n(\%)^{1}$ & $124(71.7 \%)^{x}$ & $111(52.9 \%)^{\mathrm{y}}$ & $41(61.2 \%)^{\mathrm{z}}$ & $55(53.9 \%)^{\mathrm{y}}$ & $331(60 \%)$ & 0.001 \\
\hline Beta blocker, $\mathrm{n}(\%)^{1}$ & $62(35.8 \%)^{x}$ & $134(63.8 \%)^{y}$ & $36(53.7 \%)^{\mathrm{z}}$ & $60(58.8 \%)^{\mathrm{z}}$ & $292(52.9 \%)$ & $<0.001$ \\
\hline $\begin{array}{l}\mathrm{Ca} \text {-channel blocker, } \mathrm{n} \\
(\%)^{1}\end{array}$ & $26(15 \%)^{x}$ & $71(33.8 \%)^{\mathrm{y}}$ & $18(26.9 \%)^{\mathrm{z}}$ & $28(27.5 \%)^{\mathrm{z}}$ & $143(25.9 \%)$ & 0.001 \\
\hline ACE I/ARB, n (\%) & $62(35.8 \%)^{\mathrm{x}}$ & $134(63.8 \%)^{y}$ & $36(53.7 \%)^{\mathrm{z}}$ & $60(58.8 \%)^{y}$ & $292(52.9 \%)$ & $<0.001$ \\
\hline Diuretic, $\mathrm{n}(\%)^{1}$ & $40(23.1 \%)^{x}$ & $95(45.2 \%)^{y}$ & $26(38.8 \%)^{\mathrm{z}}$ & $43(42.2 \%)^{\mathrm{y}}$ & $204(37 \%)$ & $<0.001$ \\
\hline $\begin{array}{l}24 \text { hours mean SBP } \\
(\mathrm{mmHg})^{2}\end{array}$ & $\begin{array}{c}115 \\
(90-132)\end{array}$ & $\begin{array}{c}137.5 \\
(107-184)\end{array}$ & $\begin{array}{c}135 \\
(111-189)\end{array}$ & $\begin{array}{c}136,5 \\
(111-169)\end{array}$ & $\begin{array}{c}123 \\
(90-189)\end{array}$ & $<0.001^{\mathrm{a}, \mathrm{b}, \mathrm{c}}$ \\
\hline $\begin{array}{l}24 \text { hours mean DBP } \\
(\mathrm{mmHg})^{2}\end{array}$ & $\begin{array}{c}69 \\
(56-83)\end{array}$ & $\begin{array}{c}81.5 \\
(61-122)\end{array}$ & $\begin{array}{c}81 \\
(52-109)\end{array}$ & $\begin{array}{c}83 \\
(56-105)\end{array}$ & $\begin{array}{c}74 \\
(52-122)\end{array}$ & $<0.001^{\mathrm{a}, \mathrm{b}, \mathrm{c}}$ \\
\hline $\begin{array}{l}\text { Daytime mean SBP } \\
(\mathrm{mmHg})^{2}\end{array}$ & $\begin{array}{c}117 \\
(90-193)\end{array}$ & $\begin{array}{c}137 \\
(107-188)\end{array}$ & $\begin{array}{c}143 \\
(115-193)\end{array}$ & $\begin{array}{c}140,5 \\
(116-173)\end{array}$ & $\begin{array}{c}125 \\
(90-193)\end{array}$ & $<0.001^{\mathrm{a}, \mathrm{b}, \mathrm{c}, \mathrm{d}, \mathrm{d}, \mathrm{e}, \mathrm{f}}$ \\
\hline $\begin{array}{l}\text { Daytime mean DBP } \\
(\mathrm{mmHg})^{2}\end{array}$ & $\begin{array}{c}71 \\
(55-87)\end{array}$ & $\begin{array}{c}83 \\
(60-124)\end{array}$ & $\begin{array}{c}88 \\
(56-122)\end{array}$ & $\begin{array}{c}87 \\
(58-111)\end{array}$ & $\begin{array}{c}76 \\
(55-124)\end{array}$ & $<0.001^{\mathrm{a}, \mathrm{b}, \mathrm{b}, \mathrm{d}, \mathrm{de}, \mathrm{f}}$ \\
\hline $\begin{array}{l}\text { Night-time mean SBP } \\
(\mathrm{mmHg})^{3 \mathrm{~b}}\end{array}$ & $109.4 \pm 8.6$ & $139.5 \pm 17$ & $123.5 \pm 16.4$ & $129.8 \pm 11.4$ & $126.3 \pm 18.7$ & $<0,001^{\text {a,b,c, }, \mathbf{d}, \mathbf{e}, \mathbf{f}}$ \\
\hline $\begin{array}{l}\text { Night-time mean DBP } \\
(\mathrm{mmHg})^{2}\end{array}$ & $\begin{array}{c}64 \\
(49-78)\end{array}$ & $\begin{array}{c}80 \\
(58-120)\end{array}$ & $\begin{array}{c}70 \\
(45-90)\end{array}$ & $\begin{array}{c}76 \\
(52-95)\end{array}$ & $\begin{array}{c}68 \\
(45-120)\end{array}$ & $<0.001^{\mathrm{a}, \mathrm{b}, \mathrm{c}, \mathrm{d}, \mathrm{d}, \mathrm{e}, \mathrm{f}}$ \\
\hline $\begin{array}{l}\text { Daytime mean arterial } \\
\text { pressure }(\mathrm{mmHg})^{2}\end{array}$ & $\begin{array}{c}87 \\
(48.67-101.67)\end{array}$ & $\begin{array}{c}100.33 \\
(79.33-145.33)\end{array}$ & $\begin{array}{c}105 \\
(91.67-145.67)\end{array}$ & $\begin{array}{c}105.15 \\
(84.7-131.7)\end{array}$ & $\begin{array}{c}93 \\
(48.67-145.67)\end{array}$ & $<0.001^{\mathrm{a}, \mathrm{b}, \mathrm{c}, \mathrm{d}, \mathrm{d}, \mathrm{e}, \mathrm{f}}$ \\
\hline $\begin{array}{l}\text { Night-time mean arterial } \\
\text { pressure }(\mathrm{mmHg})^{3 \mathrm{~b}}\end{array}$ & $79.09 \pm 5.9$ & $101.1 \pm 11.9$ & $87.5 \pm 9.1$ & $94.4 \pm 7.5$ & $91.3 \pm 13.1$ & $<0.001^{\mathrm{a}, \mathrm{b}, \mathrm{b}, \mathrm{c}, \mathrm{d}, \mathrm{e}, \mathrm{f}}$ \\
\hline $\begin{array}{l}24 \text { hour mean arterial } \\
\text { pressure }(\mathrm{mmHg})^{2}\end{array}$ & $\begin{array}{c}83.6 \\
(68.67-97)\end{array}$ & $\begin{array}{c}99 \\
(82-142)\end{array}$ & $\begin{array}{c}97 \\
(85.33-135.67)\end{array}$ & $\begin{array}{c}101.5 \\
(80.7-126.3)\end{array}$ & $\begin{array}{c}90.67 \\
(68.67-142)\end{array}$ & $<0.001^{\mathrm{a}, \mathrm{b}, \mathrm{c}}$ \\
\hline
\end{tabular}

MPV $=$ Mean platelet volume, RDW $=$ Red cell distribution width, LDL-C $=$ Low density lipoprotein cholesterol, HDL-C $=$ High density lipoprotein cholesterol, $\mathrm{CAD}=$ Coronary artery disease, $\mathrm{ACEI}=$ Angiotensin converting enzyme inhibitor $\mathrm{ARB}=$ Angiotensin receptor blocker, SBP $=$ Systolic blood pressure, DBP $=$ Diastolic blood pressure. Group $1=$ normotensives, Group $2=$ non-dippers, Group $3=$ extreme dippers, Group 4 = dippers

${ }^{\mathrm{a}} p<0.05$ in subgroup analysis compare to Group 1 and Group $2,{ }^{\mathrm{b}} p<0.05$ in subgroup analysis compare to Group 1 and Group 3 , ${ }^{\mathrm{c}}: p<0.05$ in subgroup analysis compare to Group 1 and Group $4,{ }^{\mathrm{d}} p<0.05$ in subgroup analysis compare to Group 2 and Group 3, ${ }^{\mathrm{e}}: p<0.05$ in subgroup analysis compare to Group 2 and Group 4, ${ }^{\mathrm{f}}: p<0.05$ in subgroup analysis compare to Group 3 and Group 4.

Each subscript letter ((shown as $(x, y, z))$ denotes a subset of grup categories whose column proportions do not differ significantly from each other at the 0.05 level in Chi square subgroup analysis. Bonferroni correction was made in subgroup analysis of parameters with kruskal wallis test. $p$ value $<0.008$ was considered significant. ${ }^{1}$ Chi square test(percentage), ${ }^{2}$ Kruskal Wallis (median (minimum-maximum)), ${ }^{3}$ One Way Anova (mean \pm standard deviation). ${ }^{3 a}$; post hoc analysis with Tukey test, ${ }^{3 \mathrm{~b}}$; post hoc analysis with Tamhane test

creased cytokine levels, especially in atherosclerosis and hypertension may have caused an increase in RDW levels by suppressing erythrocyte production in the bone marrow. Also increased sympathetic activity and angiotensin II levels, especially in non-dipper patients can stimulate the proliferation of erythrocyte progenitors and increase the RDW range [15]. In our study, we found higher RDW levels in non-dipper patients compared to the control group and the other two groups.

Mean platelet volume is the quantitative indicator of mean platelet size. Platelets are small, seedless, 


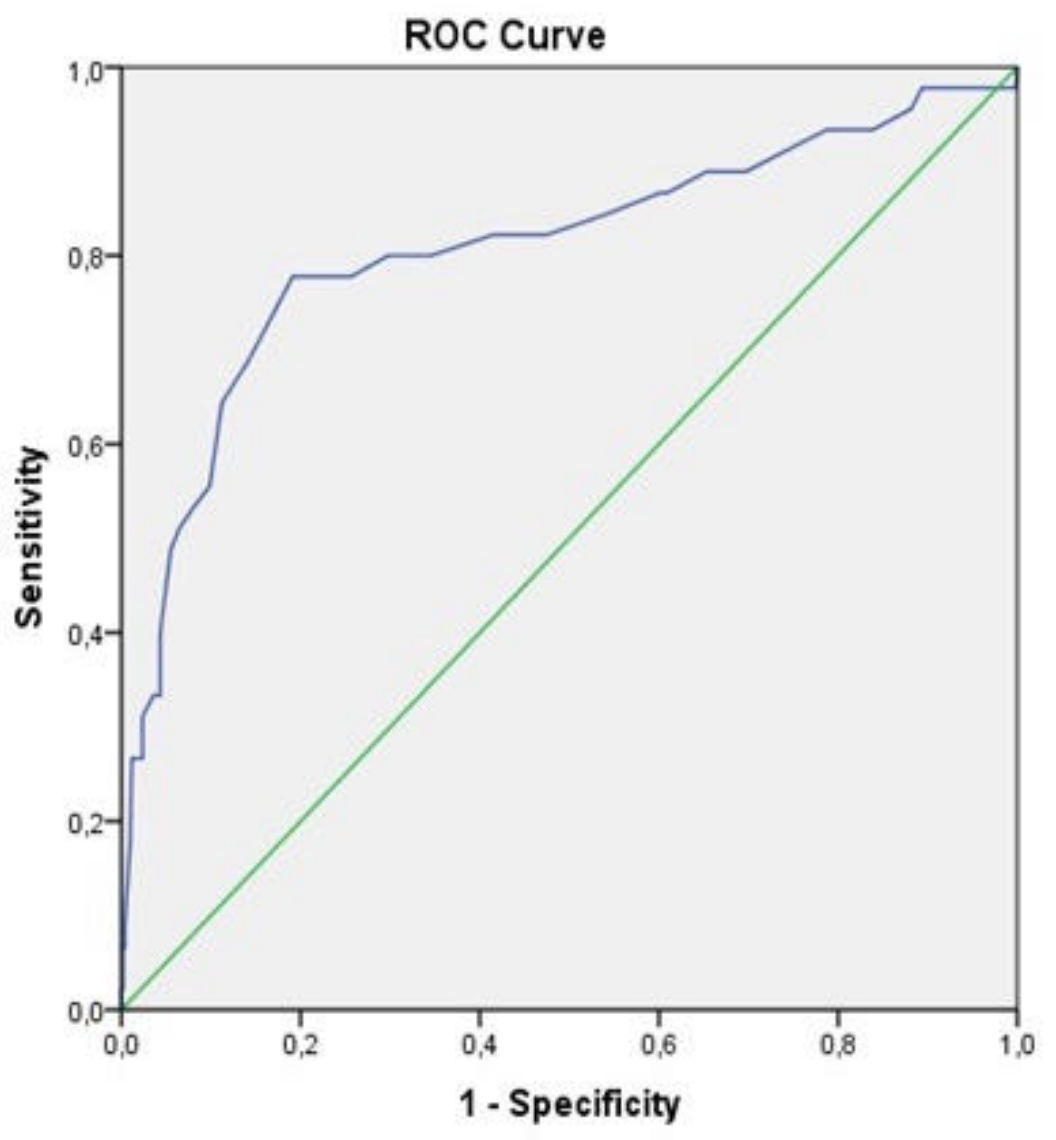

Diagonal segments are produced by ties.

Fig. 1. ROC (Receiver operation characteristic) curve and AUC (Area under the curve) for mean platelet volume for predicting stroke in hypertensive patients (Cut off: 9.25 , AUC: $0.808,95 \%$ CI:0,726- 0.889, p $<0.001,77.8 \%$ sensitivity and 78.1\% specificity).

round-shaped specialized blood cells $2-4 \mu \mathrm{m}$ in diameter. Large platelets are metabolically and enzymatically more active than small ones, which increases the susceptibility to thrombosis. As the MPV increases, the expression of glycoprotein $\mathrm{Ib}$ and glycoprotein IIb/IIIa receptors on its surface increases. Large platelets have denser granules where more proagretory factors $[16,17]$. In our study, we found higher MPV levels in non-dipper patients compared to the control group and the other two groups. Stroke and coronary artery disease rates were again higher in the non-dipper group.

In studies conducted with healthy volunteers, platelet aggregation has been shown to increase in the morning hours of the day [18]. This situation increases the risk of acute myocardial infarction in non-dipper patients with increased MPV levels. An increase in BP has been closely associated with mortality in ischemic events. Moreover, every $20 \mathrm{mmHg}$ increase in SBP and every $10 \mathrm{mmHg}$ increase in DBP increase the mortality rate two-fold indicated that the development of atherosclerotic plaque increased in non-dipper hypertensive patients and high BP at night caused endothelial damage [19].

In various studies, extreme dipper and dipper hypertensives were found to be similar in terms of cardiovascular prognosis [20]. However, different studies are documenting a better or worse prognosis in extreme dipper patients. The extreme dipper pattern ranges from $5 \%$ to $30 \%$ depending on the diagnostic criteria, clinical and demographic characteristics of the individuals [20]. In a study by Ohkubo et al. [21], the mortality risk was highest in reverse dippers, followed by non dippers; the lowest risk was associated with extreme dipping and dipper patients. In subgroup analysis, there was no difference between dipper and extreme dipper groups [21]. Additionally, a metaanalysis of several studies providing data on echocardiographic left ventricular hypertrophy indicates that extreme dippers have a lower risk of subclinical car- 
diac injury than dippers and reverse dippers [22].

Evidence of the relationship between symptomatic or silent cerebral ischemic lesions assessed by imaging techniques and nocturnal $\mathrm{BP}$ reduction is limited to a few studies in elderly hypertensive patients [22]. Severe nighttime BP reduction leading to cerebral hypoperfusion possibly related to antihypertensive therapy. However, the presence of an extreme dipper pattern, potentially harmful to cerebral perfusion, should be excluded in treated elderly hypertensive patients (especially if taking antihypertensive drugs in the afternoon or evening). However, data from the large database suggest that extreme dippers tend to be younger, less frequently obese, diabetic, or affected by cardiovascular disease. In our study, stroke rates were the highest in the non-dipper group [22]. In our study, it was similar in the extreme dipper, dipper, and normotensive groups. Coronary artery disease rates were highest in the non-dipper group. This ratio was similar in extreme dipper and dipper groups.

\section{Limitations}

The fact that our study was planned in a single center and the low number of patients participating in the study limits the power of our findings. In addition, in our study, we did not include patients with advanced heart failure, secondary hypertension, new heart attack, or valvular heart disease. Therefore, the results of our study cannot be attributed to all hypertensive patient groups.

\section{CONCLUSION}

In our study, we found higher levels of MPV and RDW showing platelet activation in the non-dipper group compared to the other groups. Also, the frequency of cerebrovascular and coronary artery disease was higher in the non-dipper group. In addition, as mentioned in some studies, we found the frequency of cerebrovascular and coronary artery disease, which is claimed to be more common in extreme dipper patients, to be similar to the dipper group.

\section{Authors' Contribution}

Study Conception: CA, ŞA; Study Design: CA, ŞA; Supervision: CA, ŞA, ME, ŞY; Funding: CA, ŞA; Materials: CA, ŞA; Data Collection and/or Processing:
CA, ŞA; Statistical Analysis and/or Data Interpretation: CA, ŞA, ME, ŞY; Literature Review: CA, ŞA, ME, ŞY; CA, ŞA, ME, ŞY: AAP and Critical Review: CA, ŞA, ME, ŞY.

\section{Conflict of interest}

The authors disclosed no conflict of interest during the preparation or publication of this manuscript.

\section{Financing}

The authors disclosed that they did not receive any grant during the conduction or writing of this study.

\section{REFERENCES}

1. Özünal ZG, Tahirbegolli İA, Baykal M, Ateş B, Tahirbegolli B, Kilıç Y, et al. The drug adherence and lifestyle factors that contribute to blood pressure control among hypertensive patients. Eur Res J 2019;5:853-60.

2. Gu JW, Liu JH, Xiao HN, Yang YF, Dong WJ, Zhang QB, et al. Relationship between plasma levels of 25-hydroxyvitamin D and arterial stiffness in elderly Chinese with non-dipper hypertension: an observational study. Medicine (Baltimore). 2020;99:e19200.

3. Kario K, Pickering TG, Umeda Y, Hoshide S, Hoshide Y, Morinari $\mathrm{M}$, et al. Morning surge in blood pressure as a predictor of silent and clinical cerebrovascular disease in elderly hypertensives: a prospective study. Circulation 2003;107:1401-6.

4. Allen LA, Felker GM, Mehra MR, Chiong JR, Dunlap SH, Ghali JK, et al. Validation and potential mechanisms of red cell distribution width as a prognostic marker in heart failure. J Cardiac Fail 2010;16:230-8.

5. Hampole CV, Mehrotra AK, Thenappan T, Gomberg-Maitland $\mathrm{M}$, Shah SJ. Usefulness of red cell distribution width as a prognostic marker in pulmonary hypertension. Am J Cardiol 2009;104:868-72.

6. Felker GM, Allen LA, Pocock SJ, Shaw LK, McMurray JJ, Pfeffer MA, et al. Red cell distribution width as a novel prognostic marker in heart failure: data from the CHARM program and the duke databank. J Am Coll Cardiol 2007;50:40-7.

7. Bath P, Algert C, Chapman N, Neal B. Association of mean platelet volume with risk of stroke among 3134 individuals with a history of CVD. Stroke 2004;35:622-6.

8. Akgül E, Engin M, Özyazıcıoğlu AF. Effects of mean platelet volume and platelet counts on peripheral biodegradable stent restenosis. J Surg Med. 2019;3:663-5.

9. Erdolu B, Engin M. The effect of mean platelet volume-tolymphocyte ratio on symptom onset in patients with carotid artery stenosis. Turk J Vasc Surg 2021;30:7-12.

10. Bofosa T, Miangindula B, Kusuayi G, Kiana N, Nkiama C. Effect of an adapted physical activity program on the morphological and physiological profile of hypertensive patients of University Clinics of Kinshasa. Eur Res J 2019;5:618-22. 
11. Sega R, Facchetti R, Bombelli M, Cesana G, Corrao G, Grassi $\mathrm{G}$, et al. Prognostic value of ambulatory and home blood pressures compared with office blood pressure in the general population: follow-up results from the Pressioni Arteriose Monitorate e Loro Associazioni (PAMELA) study. Circulation 2005;111:1777-83.

12. Fagard RH, Thijs L, Staessen JA, Clement DL, De Buyzere ML, De Bacquer DA. Night-day blood pressure ratio and dipping pattern as predictors of death and cardiovascular events in hypertension. J Hum Hypertens 2009;23:645-53.

13. Tonelli M, Sacks F, Arnold M, Moye L, Davis B, Pfeffer M. for the Cholesterol and Recurrent Events (CARE) Trial Investigators. Relation between red blood cell distribution width and cardiovascular event rate in people with coronary disease. Circulation 2008;117:163-8.

14. Tosu AR, Demir S, Selcuk M, Kaya Y, Akyol A, Ozdemir M, et al. Comparison of inflammatory markers in non-dipper hypertension vs. dipper hypertension and normotensive individuals: uric acid, C-reactive protein, and red blood cell distribution width readings. Postepy Kardiol Interwencyjnej 2014;10:98-103.

15. Seo SG, Lee MY, Park SH, Han JM, Lee KB, Kim H, et al. The association between red cell distribution width and incident hypertension in Korean adults. Hypertens Res 2020;43:55-61. 16. Villmann JM, Burkhardt R, Teren A, Villmann T, Thiery J,
Drogies T. Atherosclerosis, myocardial infarction and primary hemostasis: impact of platelets, von Willebrand factor and soluble glycoprotein VI. Thromb Res 2019;180:98-104.

17. Korniluk A, Koper-Lenkiewicz OM, Kamińska J, Kemona H, Dymicka-Piekarska V. Mean platelet volume (MPV): new perspectives for an old marker in the course and prognosis of inflammatory conditions. Mediators Inflamm 2019;2019:9213074.

18. Lewington S, Clarke R, Qizilbash N, Peto R, Collins R. Agespecific relevance of usual blood pressure to vascular mortality: a meta-analysis of individual data for one million adults in 61 prospective studies. Lancet 2002;360:1903-13.

19. Vasunta RL, Kesaniemi YA, Ylitalo A, Ukkola O. Nondipping pattern and carotid atherosclerosis in a middle-aged population: OPERA study. Am J Hypertens 2012;25:60-6.

20. Cuspidi C, Tadic M, Sala C, Gherbesi E, Grassi G, Mancia G. Extreme dipping: is the cardiovascular risk increased? An unsolved issue. J Hypertens 2019;37:1917-26.

21. Ohkubo T, Imai Y, Tsuji I, Watanabe N, Minami N, Kato J, et al. Relation between the nocturnal decline in blood pressure and mortality. Am J Hypertens 1997; 10:1201-7.

22. Cuspidi C, Tadic M, Sala C, Carugo S, Mancia G, Grassi G. Reverse dipping and subclinical cardiac organ damage: a metaanalysis of echocardiographic studies. J Hypertens 2021;39:150512. 